\title{
Specifics of reporting on cash flows in insurance companies
}

\author{
Snežana Knežević ${ }^{1 *}$, Aleksandra Mitrović ${ }^{2}$ Dušan Sretić ${ }^{3}$ \\ ${ }^{1}$ University of Belgrade, Faculty of Organizational Sciences \\ ${ }^{2}$ University in Kragujevac, Faculty of Hotel Management and Tourism in Vrnjačka Banja \\ ${ }^{3}$ EuroAudit, Belgrade
}

\begin{abstract}
The activity of insurance and operation of insurance companies is very important in the context of development of financial organizations that operate in the territory of many countries, including the Republic of Serbia. A report that is of crucial importance for insurance companies in the context of an adequate cash flow management is a cash flow statement. Bearing in mind the specificity of the insurance industry, as well as the risks associated with it, cash flow is the basic focus of financial management. For insurance companies, it presents an overview of cash flows that occurred during the previous accounting period. The aim of the paper is to show the importance of efficient cash flow management in insurance companies from the aspect of their planning, and to this effect, the paper provides the simulation of the internal report on cash flows that is done monthly for domestic insurance companies in the function of efficient liquidity management of the insurance company.
\end{abstract}

Keywords: insurance companies, cash flow statement, liquidity

JEL classification: M41, G22, G17

\section{Specifičnosti izveštavanja o novčanim tokovima u osiguravajućim kompanijama}

Sažetak: Delatnost osiguranja i rada osiguravajućih komanija je jako bitna u kontekstu razvoja finansijskih organizaciija koje posluju na teritoriji mnogih zemallja, pa i Republike Srbije. Izveštaj koji je od presudne važnosti za osiguravajuće kompanije u kontekstu adekvatnog upravljanja novčanim tokovima je izveštaj o tokovima gotovine. Imajući u vidu specifičnost industrije osiguranja, kao i rizike koji su sa njom u vezi, novčani tok je osnovni fokus finansijskog upravljanja. Za osiguravajuće kompanije, on predstavlja pregled tokova gotovine koji su se dogodili tokom proteklog računovodstvenog perioda. Cilj rada je da prikaže važnost efikasnog upravljanja novčanim tokovima u osiguravajućim kompanijama sa aspekta njihovog planiranja, te u tu svrhu i simulacije internog izveštaja o novčanim tokovima koji se izrađuje mesečno za domaću osiguravajuću kompaniju, u funkciji efikasnog upravljanja likvidnošću osiguravajuće kompanije.

Ključne reči: osiguravajuće kompanije, izveštaj o tokovima gotovine, likvidnost JEL klasifikacija: M41, G22, G17 


\section{Introduction}

Insurance is a method of transferring social risks from the insured to another person, i.e. an insurance company, for various dangers (Marcinko \& Hetico, 2006). Insurance is occasionally referred to as "business uncertainty". On the one hand, insurance is possible only in the presence of uncertainty, and on the other hand, insurance is provided by firms that seek to make a profit out of this (Zweifel \& Eisen, 2012).

Insurance business in the Republic of Serbia consists of insurance activities, including coinsurance, reinsurance business, insurance brokerage and insurance advisory services. According to Article 138 of the Insurance Act, insurance companies are obliged to keep accounts and prepare financial statements and annual report on their operations in accordance with the law and other regulations relating to the chart of accounts for insurance company and forms of financial reports and reports for statistical purposes.

Cash Flow Statement is a financial statement showing where cash is coming from (cash inflows) and where it goes away (cash outflows) for a particular accounting period, as well as what was the change in the cash balance during that period. The main element in the cash flow statement is cash, including cash equivalents as most liquid assets. Cash includes cash and sight deposits. Cash equivalents are short-term most liquid investments that can quickly turn into known amounts of cash and are not under the influence of a significant risk of a change in value. Enterprises hold cash equivalents to meet short-term cash obligations, not for investment or for some other purpose.

A cash flow statement is an overview of cash flows that occurred during the previous accounting period. IFRS (International Financial Reporting Standards) or GRP/US GAAP (Generally Accepted Accounting Principles) suggest three cash flow statement components: the cash provided from operating activities, cash provided from investment activities and cash provided through financing activities. Bearing the above mentioned in mind, there are three groups of company activities:

(1) Operating activities - Represent the main source of enterprise profits and therefore should be the primary source of cash.

(2) Investment activities - A profitable company aims to expand capacities or modernize the existing production capacities, which in the short term necessarily leads to cash outflows.

(3) Financing activities - The result of the funding activities should increase the cash resources of the enterprise, which can be used in the business and for the investment of the company.

The value of cash flow statement is that it can be used to assess the quality of earnings (profit) and financial flexibility. In addition, it can significantly help predict cash flows. The creation and analysis of cash flow statements provides important information on the results of cash resources management, and the ability to overcome the risk of doing business through securing the liquidity and solvency of an insurance company. The assessment of the success of cash flow management in practice is usually performed on the basis of horizontal analysis and on the basis of the ratio of net cash flows and profits. The horizontal analysis means the comparison of net cash flows of the current period in relation to previous periods.

The cash flow, the lifeblood of a company, is the primary focus of a financial director, both in managing daily finances and in planning and making strategic decisions in order to create value for shareholders. From the accounting perspective, the cash flows of a company can be summarized in the cash flow statement in three sections: operating activities, investment activities and financing activities. Bearing in mind that profit-oriented insurance companies employ the accrual accounting concept in their accounting, it is necessary to point out the 
well-known fact that it is necessary to monitor the flows of profitability and cash flows for efficient management of the assets of the insurance company. Quality forecasting of cash flows enables risk managers to determine the optimal amount of cash for short-term, midterm and long-term investments. Having in mind that investments cause a lack of liquidity, it is important for the treasurer to have the availability of reliable information so that it can immobilize funds and reconcile the maturities with the planned commitments.

Given the importance of insurance for the financial system of a country, on the one hand, and the importance of efficient cash management, on the other hand, for maintaining liquidity, this paper outlines the importance of managing cash flows in insurance companies. At the beginning, a review of literature will be presented, and after that, taking into account the significance of the simulations, the simulation of the internal cash flow statement on a monthly basis for domestic insurance companies is provided.

\section{Review of literature}

Mayers and Smith (1981) predict that conflict control costs between owners and managers are higher for mutual insurers rather than for stock insurers, as there are fewer mechanisms for monitoring and controlling mutual organization. By examining the relationship between the organizational form and the free cash flow, it has been established that mutual insurers have a higher level of free cash flow from stock insurers. Other firm-specific variables, such as size and leverage also affect the level of free cash flow for firms in the analyzed dataset.

Jensen (1986) argues that the existence of free cash flow provides managers with the ability to lose money on non-profitable investments. These unprofitable investments represent an additional cost of conflict between owners and managers.

The study of Wells et al. (1995) examines the differences in free cash flow between stock and mutual insurance in the U.S. life insurance industry and the purpose is to examine whether the organizational form affects managerial behavior in relation to the holding of a free cash flow. Maximize cash in, minimize cash out and cash availability is the lifeblood of the organization (Reider \& Heyler, 2003). Information contained in the cash flow statement can help investors, creditors and other users in assessing aspects of the financial position of the company, such as: (1) the ability of a company to create future cash flows and cash equivalents, (2) the ability of a company to pay dividends and meet its obligations, (3) the reasons for the resulting difference between net profit and net cash generated from operating activities and (4) investment and financing for a certain accounting period (Knežević et al., 2012).

According to Knežević et al. (2016), a cash flow statement is one of the key statements for a project or company and presents an overview of cash flows that is covered by an enterprise or project, and many users of financial statements in many industries even consider the cash flow statement to be a key financial statement.

Speaking of the simulation of the cash flow statement, which will, among other things, be presented in the paper, it is important to point out the work of Daykin and Hey (1991), which says that a cash flow model is proposed as a way of analyzing the uncertainty in the future development of a general insurance company. Also, a computer model is presented for the use in practical applications by actuaries advising the management of general insurance companies. Simulation methods are used to explore the consequences of uncertainty, particularly in regard to inflation and investments. Also, it should be borne in mind that the value of insurance company depends on the estimated operational cash flows, new investments and risks. 
According to Lukić (2010), the liquidity policy of insurance companies is a product of the very nature of insurance, and it refers in the first place to the fulfillment of obligations towards insured persons (compensation from insurance of property and persons), and only then to other persons (suppliers, creditors, state authorities). The insurance company lacking short-term cash will not be able to settle short-term liabilities as they mature, and in the longer term, it may also have solvency problems. Therefore, cash flows in insurance companies must be monitored through reporting, at least on a monthly basis.

\section{Simulation of cash flow statements in the practice of the Republic of Serbia}

Therefore, observing where money is generated and spent is just as important as the assessment of the liquidity coefficient, the profitability coefficient and other financial indicators. Basically, the cash flow statement prepared in our country by a direct method contains the following information:

- cash flows from operating activities,

- cash flows from investment activities,

- cash flows from financing activities,

- total changes in net cash,

- cash at the beginning of the accounting period and

- cash at the end of the accounting period.

In addition to direct, an indirect method for compiling cash flow statements can also be applied. The indirect method begins with a net gain, which is then corrected for non-cash items. The difference between the direct and the indirect method is only in the part that relates to operating flows.

However, it should be kept in mind that the positions in the cash flow statement are specific to the business activity they are related to. Thus, for example, for insurance companies:

Cash flows from operating activities include:

- Cash inflows from operating activities including: premium inflows (from insurance, coinsurance and reinsurance), inflows from participation in reimbursement (reinsurance and co-insurance) and

- Cash outflows from operating activities including: outflows from damage (from insurance, coinsurance and reinsurance), outflows from premiums (coinsurance and reinsurance, and commission on reinsurance and coinsurance).

Below is a simulation of the internal report on cash flows that is done monthly for domestic insurance companies. The example is followed by comments that point to the important points in the movement of cash flows (Figures 1, 2 and 3).

Due to the size of the report and the inability to present the entire report in one whole, the sections of the internal cash flow statement are shown in Tables 1, 2, 3, 4, 5 and 6, based on the operating activity, the investment activity, financing activity and "cash at the beginning of the day" (Cash flows of 11.12.2017 and 12.12.2017 included in 13.12.2017). 
Knežević, S. et al. - Specifics of reporting on cash flows in insurance companies Hotel and Tourism Management, 2018, Vol. 6, No. 2: 21-33.

Table 1: Simulation of a segment of the cash flow statement (01.12.2017-05.12.2017)

\begin{tabular}{|c|c|c|c|c|c|c|c|}
\hline $\begin{array}{c}\text { Type of } \\
\text { cash flow }\end{array}$ & $\begin{array}{l}\text { Inflows/ } \\
\text { Outflows } \\
\end{array}$ & Subtype & $\begin{array}{l}\text { Data } \\
\text { code }\end{array}$ & 1.12 .2017 & 2.12.2017 & 4.12.2017 & 5.12 .2017 \\
\hline \multirow{12}{*}{$\begin{array}{l}\text { Operating } \\
\text { activities }\end{array}$} & \multirow{4}{*}{$\begin{array}{l}\text { Cash } \\
\text { inflows } \\
\text { from } \\
\text { operating } \\
\text { activities }\end{array}$} & Premium inflows & 111 & 110,580 & 2,162 & 85,551 & 85,729 \\
\hline & & $\begin{array}{l}\text { Inflows from participation in the damage } \\
\text { compensation }\end{array}$ & 112 & 9,832 & & 315 & 213 \\
\hline & & Inflows from other operating income & 113 & 2,342 & 5 & 3,016 & 1,305 \\
\hline & & Inflows from extraordinary income & 114 & 506 & & 433 & 156 \\
\hline & \multirow{8}{*}{$\begin{array}{l}\text { Cash } \\
\text { outflows } \\
\text { from } \\
\text { operating } \\
\text { activities }\end{array}$} & Outflows on account of damages & 121 & 38,571 & & 37,674 & 43,092 \\
\hline & & Premium based outflows & 122 & & & 58 & 9,021 \\
\hline & & $\begin{array}{l}\text { Outflow from gross wages, salaries, and } \\
\text { other personal expenses }\end{array}$ & 123 & 10 & & 2 & \\
\hline & & $\begin{array}{l}\text { Outflows from taxes, contributions and } \\
\text { other taxes }\end{array}$ & 124 & 635 & & 558 & 2,491 \\
\hline & & Lease based outflows & 125 & & & & \\
\hline & & Outflow based on commissions & 126 & 5,375 & & 592 & \\
\hline & & Outflow from other operating expenses & 127 & 7,665 & 249 & 33,157 & 10,338 \\
\hline & & Outflow from other expenses & 128 & & & & \\
\hline & & $\begin{array}{l}\text { Net change in cash from operating } \\
\text { activities }\end{array}$ & & 71,004 & 1,918 & 17,274 & 22,461 \\
\hline \multirow{10}{*}{$\begin{array}{l}\text { Investing } \\
\text { activities }\end{array}$} & \multirow{5}{*}{$\begin{array}{l}\text { Inflows } \\
\text { from } \\
\text { investment } \\
\text { activities }\end{array}$} & Inflows from the sale of securities & 211 & & & & 298 \\
\hline & & Inflows from investing in securities & 212 & & & & \\
\hline & & $\begin{array}{l}\text { Inflows from sale of intangible assets and } \\
\text { fixed assets }\end{array}$ & 213 & & & & \\
\hline & & Lease inflows & 214 & & & & \\
\hline & & $\begin{array}{l}\text { Other inflows of cash from investment } \\
\text { activities }\end{array}$ & 215 & 355 & 36 & 621 & 555 \\
\hline & \multirow{6}{*}{$\begin{array}{l}\text { Outflows } \\
\text { from } \\
\text { Investing } \\
\text { activities }\end{array}$} & $\begin{array}{l}\text { Outflows from investments in securities } \\
\text { issued / guaranteed by the Republic of } \\
\text { Serbia / NBS }\end{array}$ & 221 & & & & \\
\hline & & $\begin{array}{l}\text { Outflows from investments in securities } \\
\text { traded on a regulated market }\end{array}$ & 222 & & & & \\
\hline & & $\begin{array}{l}\text { Outflows for the purchase of intangible } \\
\text { assets }\end{array}$ & 223 & & & & \\
\hline & & $\begin{array}{llll}\begin{array}{l}\text { Outflows from investments in other } \\
\text { securities }\end{array} & & \\
\end{array}$ & 224 & & & & \\
\hline & & $\begin{array}{l}\text { Other outflows of cash from investment } \\
\text { activities }\end{array}$ & 225 & & & 1,067 & 200,052 \\
\hline & & $\begin{array}{l}\text { Net change in cash from investment } \\
\text { activity }(211+212+213+214+215-221- \\
222-223-224-225)\end{array}$ & 230 & 355 & 36 & -446 & $-199,199$ \\
\hline \multirow{8}{*}{$\begin{array}{l}\text { Financing } \\
\text { activities }\end{array}$} & \multirow{4}{*}{$\begin{array}{l}\text { Inflows } \\
\text { from } \\
\text { financing } \\
\text { activities }\end{array}$} & Inflows from capital payments made & 311 & & & & \\
\hline & & Inflows from long-term loans & 312 & & & & \\
\hline & & Inflows from short-term loans & 313 & & & & \\
\hline & & Other inflows based on financing activities & 314 & & & & \\
\hline & \multirow{4}{*}{$\begin{array}{l}\text { Outflows } \\
\text { from } \\
\text { financing } \\
\text { activities }\end{array}$} & Outflows from the purchase of own shares & 321 & & & & \\
\hline & & Outflows from long-term loans & 322 & & & & \\
\hline & & Outflows from short-term loans & 323 & & & 13 & 26 \\
\hline & & Other outflows from financing activities & 324 & & & & \\
\hline & & $\begin{array}{l}\text { Net change in cash from financing } \\
\text { activities }\end{array}$ & 330 & $\mathbf{0}$ & $\mathbf{0}$ & -13 & -26 \\
\hline & & Beginning of the day cash & 500 & $1,642,181$ & $1,713,540$ & $1,715,494$ & $1,732,335$ \\
\hline
\end{tabular}

Source: Authors 
Knežević, S. et al. - Specifics of reporting on cash flows in insurance companies -

Hotel and Tourism Management, 2018, Vol. 6, No. 2: 21-33.

Table 2: Simulation of a segment of the cash flow statement (06.12.2017-09.12.2017)

\begin{tabular}{|c|c|c|c|c|c|c|c|}
\hline $\begin{array}{c}\text { Type of } \\
\text { cash flow }\end{array}$ & $\begin{array}{l}\text { Inflows/ } \\
\text { Outflows } \\
\end{array}$ & Subtype & $\begin{array}{l}\text { Data } \\
\text { code }\end{array}$ & 6.12.2017 & 7.12.2017 & 8.12.2017 & 9.12.2017 \\
\hline \multirow{12}{*}{$\begin{array}{l}\text { Operating } \\
\text { activities }\end{array}$} & \multirow{4}{*}{$\begin{array}{c}\text { Cash } \\
\text { inflows } \\
\text { from } \\
\text { operating } \\
\text { activities }\end{array}$} & Premium inflows & 111 & 88,081 & 56,347 & 93,275 & 3,326 \\
\hline & & $\begin{array}{l}\text { Inflows from participation in the damage } \\
\text { compensation }\end{array}$ & 112 & 445 & 344 & 830 & \\
\hline & & Inflows from other operating income & 113 & 1,792 & 1,612 & 1,005 & \\
\hline & & Inflows from extraordinary income & 114 & 98 & 50 & 1 & \\
\hline & \multirow{8}{*}{$\begin{array}{l}\text { Cash } \\
\text { outflows } \\
\text { from } \\
\text { operating } \\
\text { activities }\end{array}$} & Outflows on account of damages & 121 & 24,240 & 59,515 & 34,033 & \\
\hline & & Premium based outflows & 122 & & 2,353 & 283 & \\
\hline & & $\begin{array}{l}\text { Outflow from gross wages, salaries, and } \\
\text { other personal expenses }\end{array}$ & 123 & & 219 & & \\
\hline & & $\begin{array}{l}\text { Outflows from taxes, contributions and } \\
\text { other taxes }\end{array}$ & 124 & 247 & 312 & 39,568 & \\
\hline & & Lease based outflows & 125 & & & & \\
\hline & & Outflow based on commissions & 126 & 3,897 & 34 & 306 & \\
\hline & & Outflow from other operating expenses & 127 & 8,653 & 13,808 & 41,678 & 565 \\
\hline & & Outflow from other expenses & 128 & & & & \\
\hline & & $\begin{array}{l}\text { Net change in cash from operating } \\
\text { activities }\end{array}$ & & 53,379 & $-17,888$ & $-20,757$ & 2,761 \\
\hline \multirow{10}{*}{$\begin{array}{l}\text { Investing } \\
\text { activities }\end{array}$} & \multirow{5}{*}{$\begin{array}{l}\text { Inflows } \\
\text { from } \\
\text { investment } \\
\text { activities }\end{array}$} & Inflows from the sale of securities & 211 & & & & \\
\hline & & Inflows from investing in securities & 212 & & & & \\
\hline & & $\begin{array}{l}\text { Inflows from sale of intangible assets and } \\
\text { fixed assets }\end{array}$ & 213 & & & & \\
\hline & & Lease inflows & 214 & & & & \\
\hline & & $\begin{array}{l}\text { Other inflows of cash from investment } \\
\text { activities }\end{array}$ & 215 & 145 & 138 & 281 & \\
\hline & \multirow{5}{*}{$\begin{array}{l}\text { Outflows } \\
\text { from } \\
\text { Investing } \\
\text { activities }\end{array}$} & $\begin{array}{l}\text { Outflows from investments in securities } \\
\text { issued / guaranteed by the Republic of } \\
\text { Serbia / NBS }\end{array}$ & 221 & & 148,399 & 359,719 & \\
\hline & & $\begin{array}{l}\text { Outflows from investments in securities } \\
\text { traded on a regulated market }\end{array}$ & 222 & & & & \\
\hline & & $\begin{array}{l}\text { Outflows for the purchase of intangible } \\
\text { assets }\end{array}$ & 223 & & & & \\
\hline & & $\begin{array}{lllll}\begin{array}{l}\text { Outflows } \\
\text { securities }\end{array} & \text { from investments } & \text { in other } \\
\end{array}$ & 224 & & & & \\
\hline & & $\begin{array}{l}\text { Other outflows of cash from investment } \\
\text { activities }\end{array}$ & 225 & 448 & 388 & 715 & 4 \\
\hline & & $\begin{array}{l}\text { Net change in cash from investment } \\
\text { activity }(211+212+213+214+215-221-222- \\
223-224-225)\end{array}$ & 230 & 355 & -303 & $-148,649$ & $-360,153$ \\
\hline \multirow{8}{*}{$\begin{array}{l}\text { Financing } \\
\text { activities }\end{array}$} & \multirow{4}{*}{$\begin{array}{l}\text { Inflows } \\
\text { from } \\
\text { financing } \\
\text { activities }\end{array}$} & Inflows from capital payments made & 311 & & & & \\
\hline & & Inflows from long-term loans & 312 & & & & \\
\hline & & Inflows from short-term loans & 313 & & & & \\
\hline & & Other inflows based on financing activities & 314 & & & & \\
\hline & \multirow{4}{*}{$\begin{array}{l}\text { Outflows } \\
\text { from } \\
\text { financing } \\
\text { activities }\end{array}$} & Outflows from the purchase of own shares & 321 & & & & \\
\hline & & Outflows from long-term loans & 322 & & & & \\
\hline & & Outflows from short-term loans & 323 & & & & \\
\hline & & Other outflows from financing activities & 324 & & & & \\
\hline & & $\begin{array}{l}\text { Net change in cash from financing } \\
\text { activities }\end{array}$ & 330 & $\mathbf{0}$ & $\mathbf{0}$ & $\mathbf{0}$ & $\mathbf{0}$ \\
\hline & & Beginning of the day cash & 500 & $1,642,181$ & $1,552,633$ & $1,605,709$ & $1,439,112$ \\
\hline
\end{tabular}

Source: Authors 
Knežević, S. et al. - Specifics of reporting on cash flows in insurance companies Hotel and Tourism Management, 2018, Vol. 6, No. 2: 21-33.

Table 3: Simulation of a segment of the cash flow statement (13.12.2017-16.12.2017)

\begin{tabular}{|c|c|c|c|c|c|c|c|}
\hline $\begin{array}{c}\text { Type of } \\
\text { cash flow }\end{array}$ & $\begin{array}{l}\text { Inflows/ } \\
\text { Outflows } \\
\end{array}$ & Subtype & $\begin{array}{l}\text { Data } \\
\text { code }\end{array}$ & 13.12.2017 & 14.12.2017 & 15.12.2017 & 16.12.2017 \\
\hline \multirow{12}{*}{$\begin{array}{l}\text { Operating } \\
\text { activities }\end{array}$} & \multirow{4}{*}{$\begin{array}{c}\text { Cash } \\
\text { inflows } \\
\text { from } \\
\text { operating } \\
\text { activities }\end{array}$} & Premium inflows & 111 & 50,874 & 65,889 & 77,997 & 1,888 \\
\hline & & $\begin{array}{l}\text { Inflows from participation in the } \\
\text { damage compensation }\end{array}$ & 112 & 1,361 & 922 & 593 & \\
\hline & & Inflows from other operating income & 113 & 1,328 & 1,570 & 2,755 & 10 \\
\hline & & Inflows from extraordinary income & 114 & 163 & 446 & 12,460 & \\
\hline & \multirow{8}{*}{$\begin{array}{c}\text { Cash } \\
\text { outflows } \\
\text { from } \\
\text { operating } \\
\text { activities }\end{array}$} & Outflows on account of damages & 121 & 35,782 & 44,153 & 39,105 & \\
\hline & & Premium based outflows & 122 & 64,928 & 130 & 3,927 & \\
\hline & & $\begin{array}{l}\text { Outflow from gross wages, salaries, } \\
\text { and other personal expenses }\end{array}$ & 123 & & & & \\
\hline & & $\begin{array}{l}\text { Outflows from taxes, contributions and } \\
\text { other taxes }\end{array}$ & 124 & 270 & 638 & 530 & \\
\hline & & Lease based outflows & 125 & & & & \\
\hline & & Outflow based on commissions & 126 & 119 & 931 & 302 & \\
\hline & & Outflow from other operating expenses & 127 & 23,512 & 10,907 & 34,619 & 169 \\
\hline & & Outflow from other expenses & 128 & & & & \\
\hline & & $\begin{array}{l}\text { Net change in cash from operating } \\
\text { activities }\end{array}$ & & $-70,885$ & 12,068 & 15,322 & 1,729 \\
\hline \multirow{10}{*}{$\begin{array}{l}\text { Investing } \\
\text { activities }\end{array}$} & \multirow{5}{*}{$\begin{array}{l}\text { Inflows } \\
\text { from } \\
\text { investment } \\
\text { activities }\end{array}$} & Inflows from the sale of securities & 211 & & & & \\
\hline & & Inflows from investing in securities & 212 & & & & \\
\hline & & $\begin{array}{l}\text { Inflows from sale of intangible assets } \\
\text { and fixed assets }\end{array}$ & 213 & & & & \\
\hline & & Lease inflows & 214 & & & & \\
\hline & & $\begin{array}{l}\text { Other inflows of cash from investment } \\
\text { activities }\end{array}$ & 215 & 89 & 84 & 1,098 & 41 \\
\hline & \multirow{5}{*}{$\begin{array}{l}\text { Outflows } \\
\text { from } \\
\text { Investing } \\
\text { activities }\end{array}$} & $\begin{array}{l}\text { Outflows from investments in } \\
\text { securities issued / guaranteed by the } \\
\text { Republic of Serbia / NBS }\end{array}$ & 221 & & & & \\
\hline & & $\begin{array}{l}\text { Outflows from investments in } \\
\text { securities traded on a regulated market }\end{array}$ & 222 & & & & \\
\hline & & $\begin{array}{llll}\begin{array}{l}\text { Outflows for the purchase of } \\
\text { intangible assets }\end{array} & & \\
\end{array}$ & 223 & & & & \\
\hline & & $\begin{array}{l}\text { Outflows from investments in other } \\
\text { securities }\end{array}$ & 224 & & & 14 & \\
\hline & & $\begin{array}{l}\begin{array}{l}\text { Other outflows of cash from } \\
\text { investment activities }\end{array} \\
\end{array}$ & 225 & 2,262 & & 528 & \\
\hline & & $\begin{array}{l}\text { Net change in cash from investment } \\
\text { activity }(211+212+213+214+215-221- \\
222-223-224-225)\end{array}$ & 230 & 355 & $-2,173$ & 84 & 556 \\
\hline \multirow{8}{*}{$\begin{array}{l}\text { Financing } \\
\text { activities }\end{array}$} & \multirow{4}{*}{$\begin{array}{l}\text { Inflows } \\
\text { from } \\
\text { financing } \\
\text { activities }\end{array}$} & Inflows from capital payments made & 311 & & & & \\
\hline & & Inflows from long-term loans & 312 & & & & \\
\hline & & Inflows from short-term loans & 313 & & & & \\
\hline & & $\begin{array}{l}\text { Other inflows based on financing } \\
\text { activities }\end{array}$ & 314 & & & & \\
\hline & \multirow{4}{*}{$\begin{array}{l}\text { Outflows } \\
\text { from } \\
\text { financing } \\
\text { activities }\end{array}$} & $\begin{array}{l}\text { Outflows from the purchase of own } \\
\text { shares }\end{array}$ & 321 & & & & \\
\hline & & Outflows from long-term loans & 322 & & & & \\
\hline & & Outflows from short-term loans & 323 & & & & \\
\hline & & $\begin{array}{l}\begin{array}{l}\text { Other outflows } \\
\text { activities }\end{array} \\
\text { actom }\end{array}$ & 324 & & & & \\
\hline & & $\begin{array}{l}\text { Net change in cash from financing } \\
\text { activities }\end{array}$ & 330 & $\mathbf{0}$ & $\mathbf{0}$ & $\mathbf{0}$ & $\mathbf{0}$ \\
\hline & & Beginning of the day cash & 500 & $1,642,181$ & 50,874 & 65,889 & 77,997 \\
\hline
\end{tabular}

Source: Authors 
Knežević, S. et al. - Specifics of reporting on cash flows in insurance companies -

Hotel and Tourism Management, 2018, Vol. 6, No. 2: 21-33.

Table 4: Simulation of a segment of the cash flow statement (18.12.2017-21.12.2017)

\begin{tabular}{|c|c|c|c|c|c|c|c|}
\hline $\begin{array}{c}\text { Type of } \\
\text { cash flow }\end{array}$ & $\begin{array}{l}\text { Inflows/ } \\
\text { Outflows }\end{array}$ & Subtype & $\begin{array}{l}\text { Data } \\
\text { code }\end{array}$ & 18.12.2017 & 19.12.2017 & 20.12.2017 & 21.12.2017 \\
\hline \multirow{12}{*}{$\begin{array}{l}\text { Operating } \\
\text { activities }\end{array}$} & \multirow{4}{*}{$\begin{array}{c}\text { Cash inflows } \\
\text { from } \\
\text { operating } \\
\text { activities }\end{array}$} & Premium inflows & 111 & 83,932 & 63,718 & 50,816 & 64,272 \\
\hline & & $\begin{array}{l}\text { Inflows from participation in the } \\
\text { damage compensation }\end{array}$ & 112 & 17,404 & 458 & 388 & 300 \\
\hline & & Inflows from other operating income & 113 & 2,337 & 1,759 & 1,388 & 13,598 \\
\hline & & Inflows from extraordinary income & 114 & 107 & 1 & 1 & 115 \\
\hline & \multirow{8}{*}{$\begin{array}{l}\text { Cash } \\
\text { outflows } \\
\text { from } \\
\text { operating } \\
\text { activities }\end{array}$} & Outflows on account of damages & 121 & 51,597 & 22,251 & 12,590 & 53,559 \\
\hline & & Premium based outflows & 122 & 3,212 & & 4,590 & 49 \\
\hline & & $\begin{array}{l}\text { Outflow from gross wages, salaries, } \\
\text { and other personal expenses }\end{array}$ & 123 & & & & \\
\hline & & $\begin{array}{l}\text { Outflows from taxes, contributions and } \\
\text { other taxes }\end{array}$ & 124 & 192 & 91 & 29,001 & 39 \\
\hline & & Lease based outflows & 125 & & & & \\
\hline & & Outflow based on commissions & 126 & 50 & 952 & 3,890 & 1,051 \\
\hline & & Outflow from other operating expenses & 127 & 8,414 & 14,646 & 8,036 & 6,936 \\
\hline & & Outflow from other expenses & 128 & & & & \\
\hline & & $\begin{array}{l}\text { Net change in cash from operating } \\
\text { activities }\end{array}$ & & 40,315 & 27,996 & $-5,514$ & 16,651 \\
\hline \multirow{10}{*}{$\begin{array}{l}\text { Investing } \\
\text { activities }\end{array}$} & \multirow{5}{*}{$\begin{array}{l}\text { Inflows from } \\
\text { investment } \\
\text { activities }\end{array}$} & Inflows from the sale of securities & 211 & & & & \\
\hline & & Inflows from investing in securities & 212 & & & & \\
\hline & & $\begin{array}{l}\text { Inflows from sale of intangible assets } \\
\text { and fixed assets }\end{array}$ & 213 & & & & \\
\hline & & Lease inflows & 214 & & & & \\
\hline & & $\begin{array}{l}\text { Other inflows of cash from investment } \\
\text { activities }\end{array}$ & 215 & & 128 & 81 & 1,452 \\
\hline & \multirow{5}{*}{$\begin{array}{l}\text { Outflows } \\
\text { from } \\
\text { Investing } \\
\text { activities }\end{array}$} & $\begin{array}{l}\text { Outflows from investments in } \\
\text { securities issued / guaranteed by the } \\
\text { Republic of Serbia / NBS }\end{array}$ & 221 & & & & \\
\hline & & $\begin{array}{l}\text { Outflows from investments in } \\
\text { securities traded on a regulated market }\end{array}$ & 222 & & & & \\
\hline & & $\begin{array}{l}\text { Outflows for the purchase of intangible } \\
\text { assets }\end{array}$ & 223 & & & & \\
\hline & & $\begin{array}{l}\text { Outflows from investments in other } \\
\text { securities }\end{array}$ & 224 & & & & \\
\hline & & $\begin{array}{llll}\begin{array}{l}\text { Other outflows of cash from } \\
\text { investment activities }\end{array} & & \\
\end{array}$ & 225 & 105,545 & 378 & & 1,000 \\
\hline & & $\begin{array}{l}\text { Net change in cash from investment } \\
\text { activity }(211+212+213+214+215-221- \\
222-223-224-225)\end{array}$ & 230 & 355 & $-105,545$ & -250 & 81 \\
\hline \multirow{8}{*}{$\begin{array}{l}\text { Financing } \\
\text { activities }\end{array}$} & \multirow{4}{*}{$\begin{array}{l}\text { Inflows from } \\
\text { financing } \\
\text { activities }\end{array}$} & Inflows from capital payments made & 311 & & & & \\
\hline & & Inflows from long-term loans & 312 & & & & \\
\hline & & Inflows from short-term loans & 313 & & & & \\
\hline & & $\begin{array}{l}\text { Other inflows based on financing } \\
\text { activities }\end{array}$ & 314 & & & & \\
\hline & \multirow{4}{*}{$\begin{array}{l}\text { Outflows } \\
\text { from } \\
\text { financing } \\
\text { activities }\end{array}$} & $\begin{array}{l}\text { Outflows from the purchase of own } \\
\text { shares }\end{array}$ & 321 & & & & \\
\hline & & Outflows from long-term loans & 322 & & & & \\
\hline & & Outflows from short-term loans & 323 & & & & \\
\hline & & $\begin{array}{lll}\begin{array}{l}\text { Other outflows } \\
\text { activities }\end{array} & \text { from } & \text { financing } \\
\end{array}$ & 324 & & & & \\
\hline & & $\begin{array}{l}\text { Net change in cash from financing } \\
\text { activities }\end{array}$ & 330 & $\mathbf{0}$ & $\mathbf{0}$ & $\mathbf{0}$ & $\mathbf{0}$ \\
\hline & & Beginning of the day cash & 500 & $1,642,181$ & $1,059,404$ & $2,748,703$ & $2,775,909$ \\
\hline
\end{tabular}

Source: Authors 
Knežević, S. et al. - Specifics of reporting on cash flows in insurance companies Hotel and Tourism Management, 2018, Vol. 6, No. 2: 21-33.

Table 5: Simulation of a segment of the cash flow statement (22.12.2017-26.12.2017)

\begin{tabular}{|c|c|c|c|c|c|c|c|}
\hline $\begin{array}{c}\text { Type of } \\
\text { cash flow }\end{array}$ & $\begin{array}{l}\text { Inflows/ } \\
\text { Outflows }\end{array}$ & Subtype & $\begin{array}{l}\text { Data } \\
\text { code }\end{array}$ & 22.12.2017 & 23.12.2017 & 25.12.2017 & 26.12.2017 \\
\hline \multirow{13}{*}{$\begin{array}{l}\text { Operating } \\
\text { activities }\end{array}$} & \multirow{4}{*}{$\begin{array}{l}\text { Cash } \\
\text { inflows } \\
\text { from } \\
\text { operating } \\
\text { activities }\end{array}$} & Premium inflows & 111 & 74,245 & 2,688 & 131,134 & 62,332 \\
\hline & & $\begin{array}{l}\text { Inflows from participation in the damage } \\
\text { compensation }\end{array}$ & 112 & 1,940 & & 221 & 8,729 \\
\hline & & Inflows from other operating income & 113 & 6,160 & & 2,547 & 1,799 \\
\hline & & Inflows from extraordinary income & 114 & 121 & & 52 & 2 \\
\hline & \multirow{8}{*}{$\begin{array}{l}\text { Cash } \\
\text { outflows } \\
\text { from } \\
\text { operating } \\
\text { activities }\end{array}$} & Outflows on account of damages & 121 & 31,521 & & 23,439 & 45,204 \\
\hline & & Premium based outflows & 122 & 8,899 & & 9 & 7,098 \\
\hline & & $\begin{array}{l}\text { Outflow from gross wages, salaries, and } \\
\text { other personal expenses }\end{array}$ & 123 & & & & \\
\hline & & $\begin{array}{l}\text { Outflows from taxes, contributions and } \\
\text { other taxes }\end{array}$ & 124 & 530 & & 303 & 508 \\
\hline & & Lease based outflows & 125 & & & & \\
\hline & & Outflow based on commissions & 126 & 658 & & 377 & 7,471 \\
\hline & & Outflow from other operating expenses & 127 & 9,232 & 104 & 5,330 & 9,672 \\
\hline & & Outflow from other expenses & 128 & & & & \\
\hline & & $\begin{array}{l}\text { Net change in cash from operating } \\
\text { activities }\end{array}$ & & 31,626 & 2,584 & 104,496 & 2,909 \\
\hline \multirow{10}{*}{$\begin{array}{l}\text { Investing } \\
\text { activities }\end{array}$} & \multirow{5}{*}{$\begin{array}{l}\text { Inflows } \\
\text { from } \\
\text { investment } \\
\text { activities }\end{array}$} & Inflows from the sale of securities & 211 & & & & \\
\hline & & Inflows from investing in securities & 212 & & & & \\
\hline & & $\begin{array}{l}\text { Inflows from sale of intangible assets and } \\
\text { fixed assets }\end{array}$ & 213 & & & & \\
\hline & & Lease inflows & 214 & & & & \\
\hline & & $\begin{array}{l}\text { Other inflows of cash from investment } \\
\text { activities }\end{array}$ & 215 & 262 & & 411 & \\
\hline & \multirow{5}{*}{$\begin{array}{l}\text { Outflows } \\
\text { from } \\
\text { Investing } \\
\text { activities }\end{array}$} & $\begin{array}{l}\text { Outflows from investments in securities } \\
\text { issued / guaranteed by the Republic of } \\
\text { Serbia / NBS }\end{array}$ & 221 & & & & \\
\hline & & $\begin{array}{l}\text { Outflows from investments in securities } \\
\text { traded on a regulated market }\end{array}$ & 222 & & & & \\
\hline & & $\begin{array}{l}\text { Outflows for the purchase of intangible } \\
\text { assets }\end{array}$ & 223 & & & & \\
\hline & & $\begin{array}{l}\begin{array}{l}\text { Outflows from investments in other } \\
\text { securities }\end{array} \\
\end{array}$ & 224 & & & & \\
\hline & & $\begin{array}{l}\text { Other outflows of cash from investment } \\
\text { activities }\end{array}$ & 225 & 647 & & 3,969 & 267,080 \\
\hline & & $\begin{array}{l}\text { Net change in cash from investment } \\
\text { activity }(211+212+213+214+215-221- \\
222-223-224-225)\end{array}$ & 230 & 355 & -385 & & $-3,558$ \\
\hline \multirow{8}{*}{$\begin{array}{l}\text { Financing } \\
\text { activities }\end{array}$} & \multirow{4}{*}{$\begin{array}{l}\text { Inflows } \\
\text { from } \\
\text { financing } \\
\text { activities }\end{array}$} & Inflows from capital payments made & 311 & & & & \\
\hline & & Inflows from long-term loans & 312 & & & & \\
\hline & & Inflows from short-term loans & 313 & & & & \\
\hline & & Other inflows based on financing activities & 314 & & & & \\
\hline & \multirow{4}{*}{$\begin{array}{l}\text { Outflows } \\
\text { from } \\
\text { financing } \\
\text { activities }\end{array}$} & Outflows from the purchase of own shares & 321 & & & & \\
\hline & & Outflows from long-term loans & 322 & & & & \\
\hline & & Outflows from short-term loans & 323 & & & & \\
\hline & & Other outflows from financing activities & 324 & & & & \\
\hline & & $\begin{array}{l}\text { Net change in cash from financing } \\
\text { activities }\end{array}$ & 330 & $\mathbf{0}$ & $\mathbf{0}$ & $\mathbf{0}$ & $\mathbf{0}$ \\
\hline & & Beginning of the day cash & 500 & $1,642,181$ & $1,057,414$ & $1,088,675$ & $1,091,259$ \\
\hline
\end{tabular}

Source: Authors 
Knežević, S. et al. - Specifics of reporting on cash flows in insurance companies -

Hotel and Tourism Management, 2018, Vol. 6, No. 2: 21-33.

Table 6: Simulation of a segment of the cash flow statement (27.12.2017-31.12.2017)

\begin{tabular}{|c|c|c|c|c|c|c|c|}
\hline $\begin{array}{c}\text { Type of } \\
\text { cash flow }\end{array}$ & $\begin{array}{l}\text { Inflows/ } \\
\text { Outflows } \\
\end{array}$ & Subtype & 27.12.2017 & 28.12.2017 & 29.12.2017 & 30.12.2017 & 31.12.2017 \\
\hline \multirow{12}{*}{$\begin{array}{l}\text { Operating } \\
\text { activities }\end{array}$} & \multirow{4}{*}{$\begin{array}{c}\text { Cash } \\
\text { inflows } \\
\text { from } \\
\text { operating } \\
\text { activities }\end{array}$} & Premium inflows & 84,990 & 96,410 & 106,022 & 3,967 & $1,765,027$ \\
\hline & & $\begin{array}{l}\text { Inflows from participation in the } \\
\text { damage compensation }\end{array}$ & 1,516 & 1,912 & 3,899 & & 53,331 \\
\hline & & Inflows from other operating income & 1,776 & 6,249 & 2,305 & & 61,321 \\
\hline & & Inflows from extraordinary income & 481 & 101 & 103 & & 15,401 \\
\hline & \multirow{8}{*}{$\begin{array}{l}\text { Cash } \\
\text { outflows } \\
\text { from } \\
\text { operating } \\
\text { activities }\end{array}$} & Outflows on account of damages & 65,991 & 46,689 & 2,544 & & 856,264 \\
\hline & & Premium based outflows & 1,339 & 528 & 447 & & 109,601 \\
\hline & & $\begin{array}{l}\text { Outflow from gross wages, salaries, } \\
\text { and other personal expenses }\end{array}$ & & 228,534 & & & 229,890 \\
\hline & & $\begin{array}{l}\text { Outflows from taxes, contributions } \\
\text { and other taxes }\end{array}$ & 660 & 249 & 123 & 8 & 78,331 \\
\hline & & Lease based outflows & & & & & \\
\hline & & Outflow based on commissions & 2,250 & 814 & 10,886 & 33 & 40,383 \\
\hline & & $\begin{array}{llll}\begin{array}{l}\text { Outflow } \\
\text { expenses }\end{array} & \text { from } & \text { other } & \text { operating } \\
\end{array}$ & 12,252 & 17,249 & 23,499 & 190 & 330,573 \\
\hline & & Outflow from other expenses & & & & & \\
\hline & & $\begin{array}{l}\text { Net change in cash from operating } \\
\text { activities }\end{array}$ & 6,271 & $-189,391$ & 74,830 & 3,736 & 9,617 \\
\hline \multirow{10}{*}{$\begin{array}{l}\text { Investing } \\
\text { activities }\end{array}$} & \multirow{5}{*}{$\begin{array}{l}\text { Inflows } \\
\text { from } \\
\text { investment } \\
\text { activities }\end{array}$} & Inflows from the sale of securities & & & & & 298 \\
\hline & & Inflows from investing in securities & & & & & \\
\hline & & $\begin{array}{l}\text { Inflows from sale of intangible } \\
\text { assets and fixed assets }\end{array}$ & & & & & \\
\hline & & Lease inflows & & & & & \\
\hline & & $\begin{array}{llll}\begin{array}{l}\text { Other inflows of cash from } \\
\text { investment activities }\end{array} & & \\
\end{array}$ & 715 & 384 & 967 & 392 & 8,744 \\
\hline & \multirow{6}{*}{$\begin{array}{l}\text { Outflows } \\
\text { from } \\
\text { Investing } \\
\text { activities }\end{array}$} & $\begin{array}{l}\text { Outflows from investments in } \\
\text { securities issued / guaranteed by the } \\
\text { Republic of Serbia / NBS }\end{array}$ & & & & & 508,118 \\
\hline & & $\begin{array}{l}\text { Outflows from investments in secu- } \\
\text { rities traded on a regulated market }\end{array}$ & & & & & \\
\hline & & $\begin{array}{l}\text { Outflows for the purchase of } \\
\text { intangible assets }\end{array}$ & & & & & \\
\hline & & $\begin{array}{l}\text { Outflows from investments in other } \\
\text { securities }\end{array}$ & & & & & 14 \\
\hline & & $\begin{array}{lll}\begin{array}{l}\text { Other outflows of cash from } \\
\text { investment activities }\end{array} & \\
\end{array}$ & 87 & & & & 588,378 \\
\hline & & $\begin{array}{l}\text { Net change in cash from } \\
\text { investment activity }(211+212+213+ \\
214+215-221-222-223-224-225)\end{array}$ & 628 & 384 & 967 & 392 & \\
\hline \multirow{8}{*}{$\begin{array}{c}\text { Financing } \\
\text { activities }\end{array}$} & \multirow{4}{*}{$\begin{array}{l}\text { Inflows } \\
\text { from } \\
\text { financing } \\
\text { activities }\end{array}$} & Inflows from capital payments made & & & & & \\
\hline & & Inflows from long-term loans & & & & & \\
\hline & & Inflows from short-term loans & & & & & \\
\hline & & $\begin{array}{l}\text { Other inflows based on financing } \\
\text { activities }\end{array}$ & & & & & \\
\hline & \multirow{4}{*}{$\begin{array}{l}\text { Outflows } \\
\text { from } \\
\text { financing } \\
\text { activities }\end{array}$} & $\begin{array}{l}\text { Outflows from the purchase of own } \\
\text { shares }\end{array}$ & & & & & \\
\hline & & Outflows from long-term loans & & & & & \\
\hline & & Outflows from short-term loans & & & & & \\
\hline & & $\begin{array}{lll}\begin{array}{l}\text { Other outflows } \\
\text { activities }\end{array} & \text { from financing } \\
\end{array}$ & & & & & \\
\hline & & $\begin{array}{l}\text { Net change in cash from financing } \\
\text { activities }\end{array}$ & $\mathbf{0}$ & $\mathbf{0}$ & $\mathbf{0}$ & 0 & o \\
\hline & & Beginning of the day cash & $1,194,167$ & $1,201,066$ & $1,012,059$ & $1,087,856$ & $35,804,673$ \\
\hline
\end{tabular}

Source: Authors 
Figure 1: Net cash flow from operating activities

\section{OPERATING ACTIVITIES}
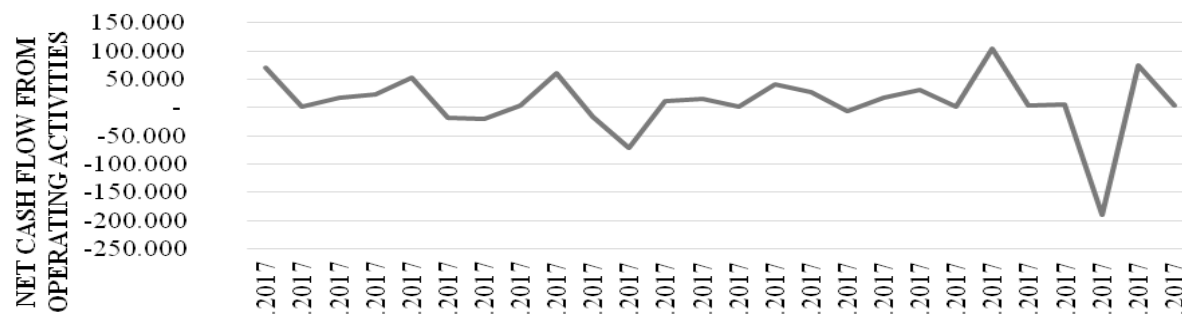

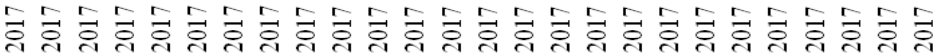

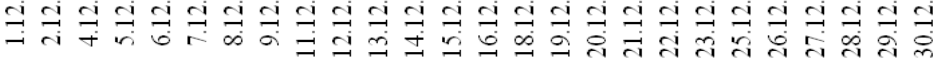
DATES

Source: Authors

Figure 2: Net changes in cash from investing activities

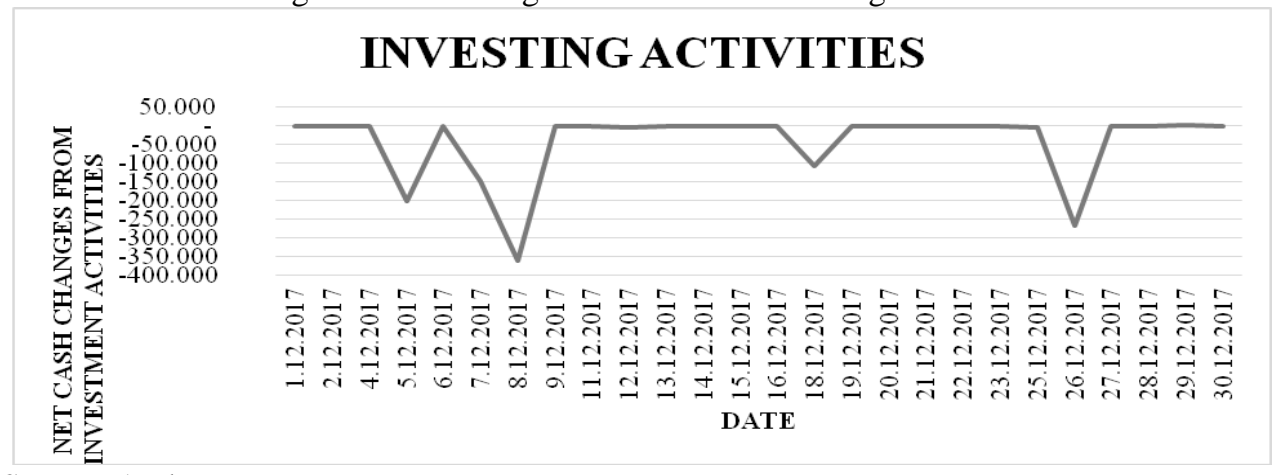

Source: Authors

Figure 3: Cash at the beginning of the day

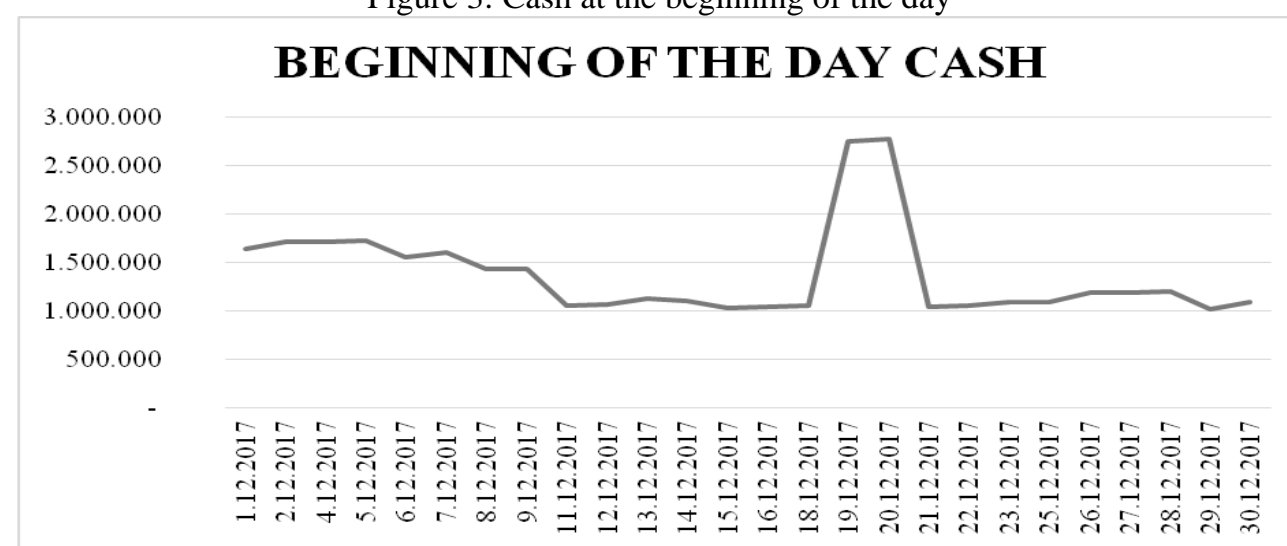

Source: Authors

1. The graph of operating activities shows that net cash changes from business activities deviated from the limit value of 9,617 m. u. until 27.12. 2017 when large cash outflows occurred. The bulk of the inflows from operating activities belongs to insurance premiums, while a large part of the outflows from operating activities are outflows from damage. 
2. The investment activities are at an even level, while large outflows of cash flows from investment activities are present, mainly related to outflows from investments in securities. Inflows from investment activities are mostly provided from income from the sale of securities.

3. Regarding monetary changes from financing activities, they did not exist, except on 4.12.2017 and 5.12.2017 when there was an outflow from short-term loans.

4. Cash at the beginning of the period has a declining trend until 16.12.2017 when it records the growth which continues until 21.12.2017. After this period, it continues to in oscillate a small degree around its main value.

\section{Conclusion}

Insurance is gaining in importance, in particular with an ever more challenging environment and increased risk, and insurance is understood as a means of risk management in a risky environment. Insurance companies, bearing in mind the foregoing, must take into account cash flows in order to lead the decisions to the achievement of objectives, and in doing so, there would be no problems with the liquidity of the insurance companies. Cash flow planning is one of the most difficult and vulnerable areas in business management, especially in the insurance industry where many risks are immanent. When talking about insurance companies, it is necessary to suggest that cash is the lifeblood of the firm and that insurance company's cash planning practices can be a critical early warning device of impending financial problems, especially in the segment of investing funds in financial investments (long-term and short-term). From a risk perspective, a particularly sensitive item is long-term financial placements.

The cash flow statement of an entity is a basic choice of information used to make operational decisions regarding the management of cash flows, to control the financial solvency of the entity, and to interpret the differences between financial results and changes in the amount of cash. Bearing in mind the significance of cash flow statements for insurance companies, it can be concluded that, in addition to using them for the assessment of the quality of earnings and financial flexibility, they can greatly assist in forecasting cash flows. In addition, they provide us with key information for assessing the quality of earnings, the degree of reproducibility of business resources, as well as the level of competitiveness in the insurance market in which the insurance company is operating.

The monthly simulation of the internal report on cash flows for domestic insurance companies is of great importance to insurance companies. Information on cash changes is useful in order to assess its investments, financial and operational activities during the accounting period. This information gives different stakeholders the basis to assess the ability of the company to generate cash and its equivalents, as well as the needs of business to use these flows appropriately. Cash management is a process of planning, controlling and accounting monitoring of cash transactions and balances in order to prevent the loss of money and maintain its adequate level.

\section{References}

1. Daykin, C. D., \& Hey, G. B. (1991). A stochastic cash-flow model of a general insurance company. In Presentado a AFIR Colloquium, 4, 113-137.

2. Jensen, M. C. (1986). Agency costs of free cash flow, corporate finance, and takeovers. The American Economic Review, 76(2), 323-329. http://dx.doi.org/10.2139/ssrn.99580 
3. Knežević, S., Dmitrović, V., Stanković, A., \& Mitrić, M. (2012). Poslovnofinansijsko odlučivanje na osnovu dobiti ili gotovine [Business financial decisionmaking based on profit or cash], 13. Međunarodna znanstvena i stručna konferencija „Računovodstvo $i$ menadžment", 14-16. jun 2012. godine, Zagreb, Hrvatski računovođa, Neovisna Udruga računovođa, poreznih savjetnika i financijskih dejelatnika, 258-270.

4. Knežević, S., Mitrović, A., \& Ilić, Z. (2016). Pogled na izveštavanje o tokovima gotovine iz različitih perspektiva [Different perspectives on the cash flow statement]. Menadžment u hotelijerstvu i turizmu, 4(2), 36-47.

5. Lukić, R. (2010). Računovodstvo osiguravajućih kompanija [Accounting of Insurance Companies], Beograd: Ekonomski fakultet, Univerzitet u Beogradu.

6. Marcinko, D. E., \& Hetico, H. R. (2006). Dictionary of health insurance and managed care. Springer Publishing Company, Inc.

7. Mayers, D., \& Smith Jr, C. W. (1981). Contractual provisions, organizational structure, and conflict control in insurance markets. Journal of Business, 54(3), 407434. http://dx.doi.org/10.1086/296138

8. Reider, R., \& Heyler, P. B. (2003). Managing cash flow: An operational focus. John Wiley \& Sons.

9. Wells, B. P., Cox, L. A., \& Gaver, K. M. (1995). Free cash flow in the life insurance industry. Journal of Risk and Insurance, 62(1), 50-66. http://dx.doi.org/10.2307/253692

10. Zakon o osiguranju, Službeni glasnik RS, br. 139/2014 [Insurance Act, Official Gazette of RS, no. 139/2014].

11. Zweifel, P., \& Eisen, R. (2012). Insurance economics. Springer Science \& Business Media. 